\title{
Effect of EGb761 on the Dynamic Changes of Energy Metabolites and Bio-Metal in the Brain Cortex of Gerbil during Focal Cerebral Ischemia
}

\author{
Ming-Cheng Lin \\ Department of Medical Laboratory Science and Biotechnology, Central Taiwan University of Sciences and \\ Technology, Taiwan \\ Email: mclin@ctust.edu.tw
}

Received 18 November 2015; accepted 3 December 2015; published 7 December 2015

Copyright (C) 2015 by author and OALib.

This work is licensed under the Creative Commons Attribution International License (CC BY). http://creativecommons.org/licenses/by/4.0/

(c) (i) Open Access

\section{Abstract}

The aim of this study was to evaluate the pharmacological effect of EGb761 on the dynamic alterations of magnesium (Mg) and energy metabolites including glucose and lactate in brain cortex of gerbil during focal cerebral ischemia. A single dose of EGb761 (100 mg/kg, i.p.) was given $30 \mathrm{~min}$ prior to cerebral ischemic surgery. Cerebral ischemia was induced by occlusion of the right middle cerebral artery (RMCA) for $\mathbf{1 8 0}$ minutes. Two microdialysis probes were stereotaxically implanted bilaterally into the brain cortex for auto-sampling the extracellular fluid. Experimental result showed that pretreatment gerbil with EGb761 could preserve the highest Mg and glucose level in the contralateral (non-ischemic) brain. However, the lowest lactate concentration was observed in the contralateral (non-ischemic) brain in gerbil pretreated with EGb761. Interestingly, an opposite result concerning the level of $\mathrm{Mg}$, glucose, and lactate was observed in the ipsilateral (ischemic) brain in EGb761 pretreated subject. Based on our present results, it seems likely that due to the pharmacological and compensatory effect, EGb761 can preserve more Mg and glucose level but not the lactate concentration in the contralateral brain. Also, this positive phenomenon, at least in part, may be helpful for the non-ischemic brain not only in preserving more Mg and glucose level, but also in preventing the non-ischemic brain from further serious cerebral ischemic challenge.

\section{Keywords}

Cerebral Ischemia, Microdialysis, Magnesium, Glucose, Lactate, EGb761

Subject Areas: Neurology, Neuroscience, Pharmacology

How to cite this paper: Lin, M.-C. (2015) Effect of EGb761 on the Dynamic Changes of Energy Metabolites and Bio-Metal in the Brain Cortex of Gerbil during Focal Cerebral Ischemia. Open Access Library Journal, 2: e2204.

http://dx.doi.org/10.4236/oalib.1102204 


\section{Introduction}

Magnesium (Mg) has been evidenced in possessing a variety of crucial roles including maintaining normal cellular functions, acting as an antagonist of the calcium ion, playing a central importance in energy metabolism, and inhibiting the calcium ion flows into the cell [1]. Conversely, a variety of human disorders have been documented in responsible for reduced Mg level [2] [3]. Ginkgo biloba leaf extract (EGb761), an ancient Chinese medicine, has been proposed for their beneficial efficacy in regulating blood circulation, enhancing blood flow into the ischemic brain, and alleviating brain swelling due to the component of flavonoid [4] [5]. Based on its broad physiological functions, EGb761 has been clinically used in Taiwan, Germany, and France for ameliorating cerebral ischemic injury [5]. It has been recognized that cerebral ischemic insult may lead to disability and death in older adults. Moreover, it has been proposed that once oxygen and energy supplementation declines to the critical levels in the brain, large amount of the increased reactive oxygen species may result in further brain lesion [6]. Glucose is the major energy source especially for the brain [7]. In contrast, lactate is the end product of glucose. Previous literature has evidenced the fact that cerebral ischemia may elevate the lactate level and is realized to be harmful to the brain tissue [8]. Microdialysis technique is an effective auto-sampling tool which is available for dynamically collecting and monitoring the metabolic status of the information from microenvironment space such as the brain [9] [10]. The aim of this present study was to experimentally investigate the effect of EGb761 on the dynamic alterations of the level of Mg, glucose, and lactate in the brain cortex of gerbils.

\section{Materials and Methods}

Groups of six male gerbils ( $\mathrm{n}=6,65$ - 85 g) were subjected and animals were allowed to acclimate to their environmentally controlled quarters $\left(25^{\circ} \mathrm{C}\right.$ and $12: 12 \mathrm{~h}$ light-dark cycle). Focal cerebral ischemia was induced by occlusion of the unilateral right middle carotid artery (RMCA) for $180 \mathrm{~min}$. Two microdialysis probes (4 mm in length, CMA/12, Carnegie Medicin, Stockholm, Sweden) were stereotaxically implanted into both sides of the brain cortex (AP $0 \mathrm{~mm}, \mathrm{ML}+/-5 \mathrm{~mm}, \mathrm{DV}-5 \mathrm{~mm}$ from bregma). EGb761 produced as the tablets of Cerenin was purchased from the company of Schwabe Karlsruhe in the Germany. A single dose of EGb761 (100 mg/kg, i.p) and saline was given 30 min prior to RMCA occlusion, individually. Dialysis probes were perfused with Ringer's solution (147 $\left.\mathrm{mM} \mathrm{Na}^{+} ; 2.2 \mathrm{mM} \mathrm{Ca}^{2+} ; 4 \mathrm{mM} \mathrm{K}^{+}, \mathrm{pH} 7.0\right)$ at a flow rate of $2 \mu \mathrm{l} / \mathrm{min}$ by CMA/100 infusion pump. Dialysates were collected every 15 min by means of a CMA/140 fraction collector (Carnegie Medicin, Stockholm, Sweden). Mg level was measured using atomic absorption spectrometry (Model Analyst 300, Perkin-Elmer, Uberlingen, Germany). Levels of glucose and lactate were enzymatically oxidized by glucose oxidase and lactate oxidase individually using microdialysis analyzer (CMA/600, Carnegie Medicin, Stockholm, Sweden). All the experimental data were expressed as mean \pm SEM. Animal use protocol has been reviewed and approved by the Institutional Animal Care and Use Committee (IACUC) of the Central Taiwan University of Sciences and Technology.

\section{Results}

In the present experiment, the analytical performance of atomic absorption spectrometry in the determination of Mg level in the extracellular fluid of the brain cortex in gerbils was listed in Table 1. Obviously, it is of note that an excellent analytical performance in the present study for the analysis of essential trace element of $\mathrm{Mg}$ is achieved.

Table 1. Analytical performance of atomic absorption spectrometry in the determination of Mg level in the extracellular fluid of the brain cortex in gerbils.

\begin{tabular}{cc}
\hline Bio-metal & Mg \\
\hline Linear range $(\mathrm{mg} / \mathrm{mL})$ & $0.50-3.50$ \\
Slope & 0.247 \\
Correlation coefficient & 0.998 \\
Detection limit $(\mathrm{mg} / \mathrm{mL})$ & 0.03 \\
Recovery $(\%)(\mathrm{n}=6)$ & 104 \\
\hline
\end{tabular}


Mg levels were declined to $60 \%$ and $80 \%$ of the baseline on ischemic side in EGb761 and saline treated groups during cerebral ischemia. The highest $\mathrm{Mg}$ level was seen on the non-ischemic brain in EGb761 treated group. Interestingly, the lowest $\mathrm{Mg}$ level was observed on non-ischemic side in EGb761 treated rats (Figure 1).

Glucose is recognized the major energy source for the brain. Data showed that the glucose levels were declined to $65 \%$ and $75 \%$ of the baseline level on the ischemic side in EGb761 and saline treated group, individually (Figure 2). In addition, on the non-ischemic side of EGb761 pretreated gerbils, glucose levels were increased to $140 \%$ of the baseline in the present experiment as showed in Figure 2.

Lactate is the end product of glucose metabolism. As listed in Figure 3, our present data showed that the lactate levels in the ischemic side in EGb761 and saline pretreated groups were declined to $60 \%$ and $80 \%$ of the baseline, respectively.

\section{Discussion}

It has been known that the brain is extremely sensitive to energy and oxygen deprivation. Once oxygen and energy supplementation is compromised, damage to the brain tissue occurs. Recently, accruing interest has focused more attention on the role of Mg in clinical medicine, neurology and nutrition [1] [2]. Mg is realized to be

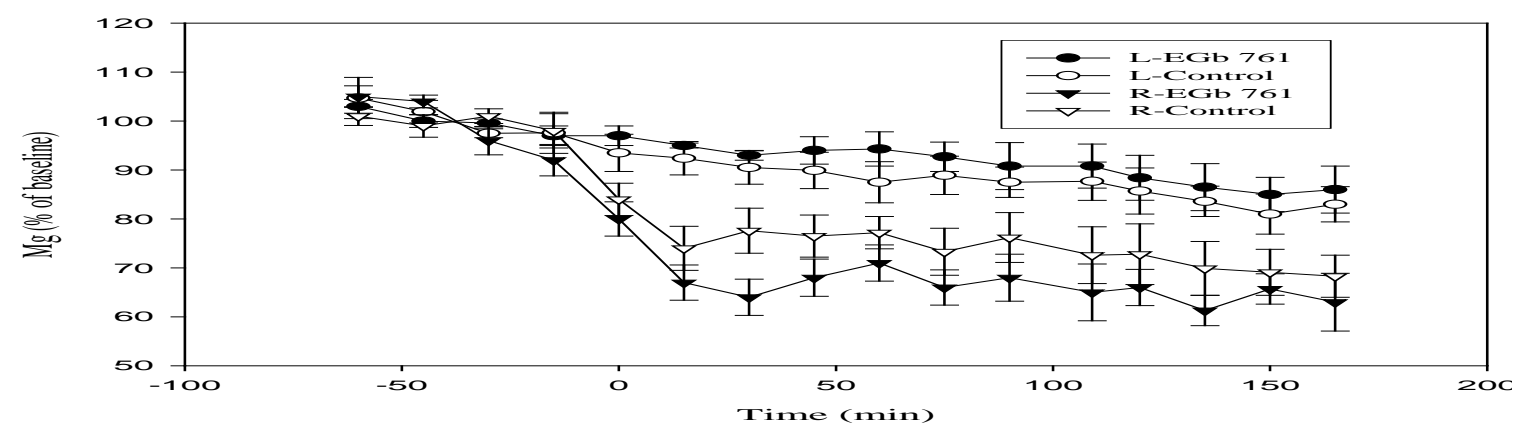

Figure 1. Profile of Mg levels on ipsilateral (ischemic) and contralateral (non-ischemic) brain during RMCA occlusion. Data are expressed as mean $\pm \operatorname{SEM}(n=6)$.

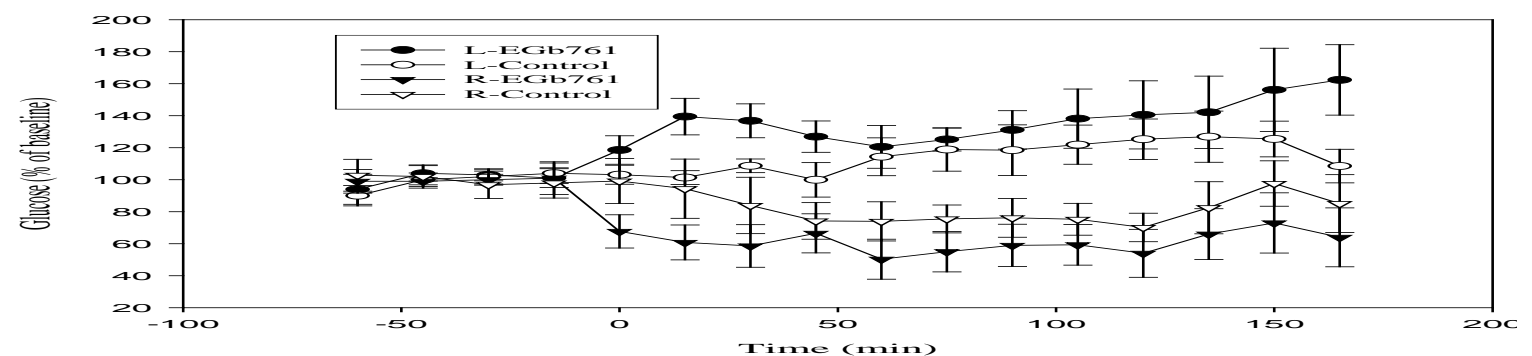

Figure 2. Dynamic profile of the glucose levels on ischemic and non-ischemic brain cortex during RMCA occlusion. Data are expressed as mean \pm SEM $(n=6)$.

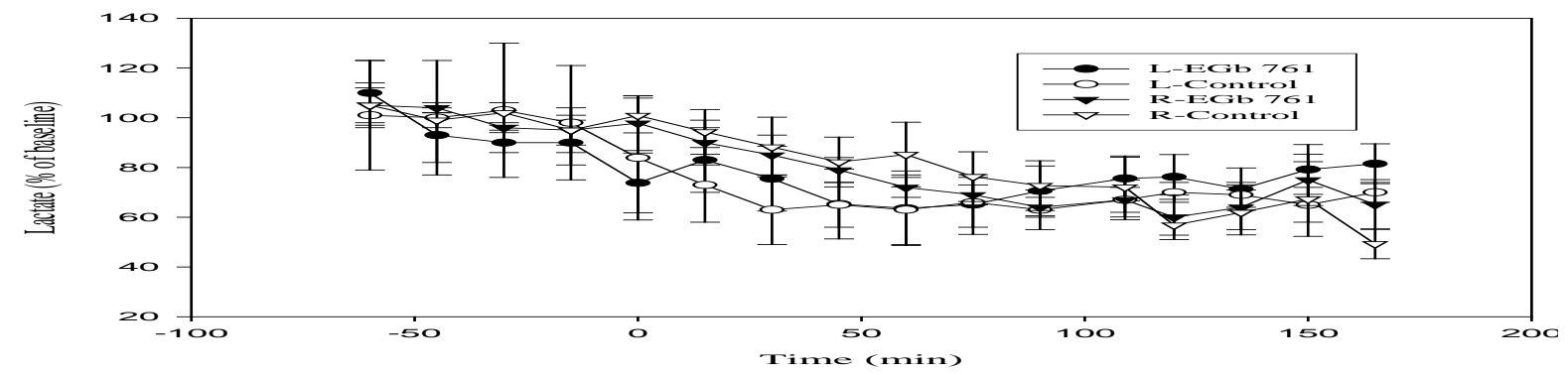

Figure 3. Profiles of lactate levels on ischemic and non-ischemic brain during RMCA occlusion. All the experimental data are expressed as mean $\pm \operatorname{SEM}(n=6)$. 
required for animals and plants. Moreover, it has been revealed that Mg is important for those enzymes which use nucleotides as a cofactors or substrates [3]. Disturbance of Mg level has been validated in responsible for a variety of human disorders [2] [3]. Similarly, our former study has revealed that a significantly declined Mg level was observed in cerebral ischemia [1]-[3]. Indeed, $\mathrm{Mg}$ is crucial for living organisms not only in decreasing calcium transportation due to its antagonistic effects, but also in retarding further biochemical cascade damage to the cells. Additionally, our former study has indicated that erythrocytes may preserve more Mg level during cerebral ischemia [3]. Study has demonstrated that elevated $\mathrm{Mg}$ level may decline the glucose utilization rate and given the fact, lactate concentration was reduced [2]. Instead, deficiency in Mg level has been reported not only in correlated with increased free radicals level but also in resulting in energy failure [2] [3]. In this current study, the Mg levels in the ipsilateral side in EGb761 and saline treated group were significantly declined to $60 \%$ and $80 \%$ of the baseline level, individually. Interestingly, the highest and the lowest Mg level were found in the contralateral and the ipsilateral side in EGb761 treated gerbils, respectively. Previous study indicated that EGb761 possessed a pharmacological effect in enhancing cerebral blood flow and dilating blood vessels [4] [8]. Additionally, it seems likely that due to the compensatory effect, more Mg level which is existed in the ischemic brain may be flow into the non-ischemic brain. This phenomenon is thinkable to be advantageous to the brain tissue. According, a possibility we infer here that pretreatment gerbils with EGb761, partially for increasing cerebral blood flow and partially for preserving more Mg level, more Mg was accumulated in the contralateral side. Conversely, declined Mg concentration was found on the ipsilateral side of the brain cortex in EGb761 treated gerbils. Cerebral ischemia has been recognized as an acute metabolic energy crisis. Dynamic change of the energy-related metabolites in brain extracellular space during ischemia is depends upon the degree of severity. Under normal situations, glucose is the major and important energy source of special for the brain cells. By means of glycolysis pathway, glucose can be catabolized and to produce the energy source of adenosine triphosphate (ATP). In this regard, keeping an appropriate glucose concentration is useful for the brain in keeping normal physiological functions. In this study, the glucose levels were markedly declined to $65 \%$ and $75 \%$ of the baseline on the ipsilateral side in EGb761 and saline treated group, individually. On the contralateral side of the EGb761 treated animals, the glucose level increased to $140 \%$ of the baseline. Further, the lowest glucose level which declined the glucose level to 65\% of the baseline was observed on the ipsilateral side in EGb761 treated group (Figure 2). Likewise, a paralleled relationship between the biochemical parameter of glucose and Mg was achieved in the present study. In fact, Mg has also been illustrated for playing a central importance in energy metabolism including glycolysis, oxidative phosphorylation, and ATPase function. Moreover, previous study [4] [8] has indicated that EGb761 possesses the ability in preserving glucose levels in the brain during cerebral ischemia, and our present result was in accordance with the previous evidence. Based on our results, it seems possible that due to its pharmacological effect, EGb761 not only may increase the cerebral blood flow into the contralateral side of the brain cortex, but also may preserve more $\mathrm{Mg}$ and glucose level in the non-ischemic brain. Possibly, this beneficial efficacy is helpful for the non-ischemic brain in preventing further critical challenge caused by the ischemic crisis. On the other hand, it is well-recognized that production of the energy metabolite of lactate during ischemia is strongly dependent on glucose supplementation. The lactate level in the ischemic brain in EGb761 and saline administrated groups was diminished to $60 \%$ and $80 \%$ of the baseline level, individually. A negative correlation between the glucose and the lactate level was found in the present study. Previous study has indicated that elevated lactate level in tissue represents acidosis or bad situations such as cerebral ischemia [11] [12]. Our experimental result indicated that pretreatment gerbils with EGb761 could preserve more glucose and decline the lactate level. This effect may be advantageous to the brain tissue.

\section{Conclusion}

In summary, a possibility we infer here that due to the pharmacological and compensatory effect, EGb761 can preserve more Mg and glucose level in the non-ischemic brain. Also, this positive phenomenon, at least in part, may be helpful for the non-ischemic brain not only in preserving more $\mathrm{Mg}$ and glucose level, but also in preventing the non-ischemic brain from further serious cerebral ischemic challenge.

\section{References}

[1] Yang, D.Y., Lee, J.B., Lin, M.C., Huang, Y.L., Liu, H.W., Liang, Y.J. and Cheng, F.C. (2004) The Determination of Brain Magnesium and Zinc Levels by a Dual-Probe Microdialysis and Graphite Furnace Atomic Absorption Spectro- 
metry. Journal of the American College of Nutrition, 23, 552S-555S.

http://dx.doi.org/10.1080/07315724.2004.10719402

[2] Fromm, L., Heath, D.L., Vink, R. and Nimmo, A.J. (2004) Magnesium Attenuates Post Traumatic Depression/Anxiety Following Diffuse Traumatic Brain Injury in Rats. Journal of the American College of Nutrition, 23, 529S-533S. http://dx.doi.org/10.1080/07315724.2004.10719396

[3] Lin, M.C., Huang, Y.L., Liu, H.W., Yang, D.Y., Lee, C.P., Yang, L.L. and Cheng, F.C. (2004) On-Line Microdialysis-Graphite Furnace Atomic Absorption Spectrometry in the Determination of Brain Magnesium Levels in Gerbils Subjected to Cerebral Ischemia/Reperfusion. Journal of the American College of Nutrition, 23, 561S-565S. http://dx.doi.org/10.1080/07315724.2004.10719404

[4] Chung, S.Y., Cheng, F.C., Lee, M.S., Lin, J.Y., Lin, M.C. and Wang, M.F. (2006) Ginkgo Biloba Leaf Extract (EGb761) Combined with Neuroprotective Agents Reduces the Infarct Volumes of Gerbil Ischemic Brain. The American Journal of Chinese Medicine, 34, 803-817. http://dx.doi.org/10.1142/S0192415X06004302

[5] Lin, M.C. (2015) EGb761 Co-Administrated with FK506 Offer No More Neuro-Protective Efficacy than EGb761 Alone during Focal Cerebral Ischemia and Reperfusion Monitored by Microdialysis Coupled with Microbore Liquid Chromatography. International Journal of Medicine and Pharmacy, 3, 41-53.

[6] Valko, M., Morris, H. and Cronin, M.T. (2005) Metals, Toxicity and Oxidative Stress. Current Medicinal Chemistry, 12, 1161-1208. http://dx.doi.org/10.2174/0929867053764635

[7] Scheufler, K.M., Lehnert, A., Rohrborn, H.J., Nadstawek, J. and Thees, C. (2004) Individual Value of Brain Tissue Oxygen Pressure, Microvascular Oxygen Saturation, Cytochrome Redox Level, and Energy Metabolites in Detecting Critically Reduced Cerebral Energy State during Acute Changes in Global Cerebral Perfusion. Journal of Neurosurgical Anesthesiology, 16, 210-219. http://dx.doi.org/10.1097/00008506-200407000-00005

[8] Urikova, A., Babusikova, E., Dobrota, D., Drgova, A., Kaplan, P., Tatarkova, Z. and Lehotsky, J. (2006) Impact of Ginkgo Biloba Extract EGb761 on Ischemia and Reperfusion Induced Oxidative Stress Products Formation in Rat Forebrain. Cellular and Molecular Neurobiology, 26, 1341-1351. http://dx.doi.org/10.1007/s10571-006-9030-3

[9] Jyranki, J., Suominen, S., Vuola, J. and Back, L. (2006) Microdialysis in Clinical Practice: Monitoring Intraoral Free Flaps. Annals of Plastic Surgery, 56, 387-393. http://dx.doi.org/10.1097/01.sap.0000205231.39853.87

[10] Poling, J., Rees, W., Klaus, S., Bahlmann, L., Hubner, N., Mantovani, V. and Warnecke, H. (2007) Myocardial Metabolic Monitoring with the Microdialysis Technique during and after Open Heart Surgery. Acta Anaesthesiologica Scandinavica, 51, 341-346. http://dx.doi.org/10.1111/j.1399-6576.2006.01241.x

[11] Lin, J.Y., Chung, S.Y., Lin, M.C. and Cheng, F.C. (2002) Effects of Magnesium Sulfate on Energy Metabolites and Glutamate in the Cortex during Focal Cerebral Ischemia and Reperfusion in the Gerbil Monitored by a Dual-Probe Microdialysis Technique. Life Sciences, 71, 803-811. http://dx.doi.org/10.1016/S0024-3205(02)01738-1

[12] Lin, M.C., Huang, Y.L., Liu, H.W., Yang, D.Y., Lee, J.B. and Cheng, F.C. (2004) Microdialysis Analyzer and Flame Atomic Absorption Spectrometry in the Determination of Blood Glucose, Lactate, and Magnesium in Gerbils Subjected to Cerebral Ischemia/Reperfusion. Journal of the American College of Nutrition, 23, 556S-560S. http://dx.doi.org/10.1080/07315724.2004.10719403 